\title{
Structure comparée des glycannes des sérotransferrines et des lactotransferrines
}

par

Geneviève SPIK et J. MONTREUIL*

\section{S u m m a r y}

The transferrins (serum transferrins and lactotransferrins) play an important role in bacteriostatic activity of the organisms and in the transport of iron into different receptor cells. The peptidic moiety of the various transferrins presents structural homologies while the primary structure of the glycans is peculiar to each species and could explain the specificity of cell recognition.

Titre abrégé

Structure des glycannes des transferrines.

Tittle

Structure of glycans from transferrins.

Key words

Glycans - Transferrin - Lactotransferrin - Lactoferrin.

* Laboratoire de Chimie biologique de l'Université des Sciences et Techniques de Lille I, et Laboratoire associé au C.N.R.S., n 217 - 59655 Villeneuve-d'Ascq cedex. 


\section{INTRODUCTION}

La forme majeure de transport du fer dans les milieux d'excrétion comme le lait, la salive, les larmes et le mucus bronchique est représentée par la lactotransferrine (LTF). Cette glycoprotéine révèle de nombreuses analogies structurales et fonctionnelles avec la sérotransferrine (STF) présente dans le sang de tous les Vertébrés et avec l'ovotransferrine (OTF) du blanc d'œuf des Oiseaux. Ainsi, par leur capacité de fixer spécifiquement et réversiblement deux ions $\mathrm{Fe}^{3+}$ et de priver de fer les milieux biologiques, ces trois transferrines possèdent une activité bactériostatique. En outre, par leurs propriétés de reconnaître des sites récepteurs membranaires spécifiques, elles assurent le transport et le passage du fer à l'intérieur de différentes cellules comme les réticulocytes pour les sérotransferrines, les entérocytes pour les lactotransferrines et les cellules embryonnaires des globules rouges du Poussin pour l'ovotransferrine.

La connaissance de la structure des fractions peptidiques et glucidiques est apparue nécessaire pour établir des relations entre structure et activité de ces trois transferrines. Les travaux récents de Mac Gillivray et al., 1982 sur la STF humaine, de Jeltsch et Chambon, 1982 et de Williams et al., 1982 sur l'OTF de Poule et de Metz-Boutigue et al., 1981 sur la LTF humaine ont montré que les séquences peptidiques des trois transferrines présentaient de nombreuses homologies.

Au laboratoire, nous nous sommes plus particulièrement intéressés à l'étude comparative de la structure des glycannes afin de définir leur rôle dans les différentes activités biologiques que présentent les trois transferrines. 


\section{STRUCTURE DES GLYCANNES DES TRANSFERRINES}

\section{Sérotransferrines}

La première détermination de structure d'un glycanne mature de type biantenné N-acétyllactosaminique disialylé (fig. 1) a été réalisée dans le cas de la STF humaine (Spik et al., 1975), Depuis, la même structure a été identifiée dans les STF de Lapin (Léger et al., 1978), de Bœuf, de Mouton et de Poule (Spik et al., résultats non publiés). Ces résultats démontrent qu'il existe une certaine unité de structure des glycannes. Toutefois, des différences existent qui concernent, d'une part, le nombre des glycannes portés par les chaînes peptidiques (deux dans la STF humaine, un dans la STF de Lapin) et, d'autre part, la présence d'une faible proportion (10 à $15 \%)$ d'un glycanne de type N-acétyllactosaminique triantenné (fig. 1) dans la STF humaine (Spik et al., 1982).

\section{Lactotransferrines}

Les structures des glycannes des lactotransferrines des laits de Femme, de Vache et de Chèvre ont été déterminées. La LTF humaine renferme 2 glycannes qui présentent une importante micro-hétérogénéité liée au nombre très variable de résidus de fucose, d'acide $\mathrm{N}$-acétylneuraminique et de fucosyl- $\mathrm{N}$-acétyllactosamine qui substituent la structure undécasaccharidique de base (fíg, 2) (Spik et al., 1982). D'autre part, les LTF de Vache et de Chèvre renferment, outre des glycannes de type $\mathrm{N}$-acétyllactosaminique, des glycannes de type oligomannosidique. Ces derniers présentent, eux aussi, une certaine hétérogénéité puisque des structures renfermant de 5 à 9 résidus de mannose ont été identifiés (Spik et al., résultats non publiés). La structure primaire complète du glycanne le plus complexe possédant 9 résidus de mannose a été déterminé (Van Halbeek et al., 1981) (fig. 3). Ces résultats montrent que les glycannes des LTF de Vache et de Chèvre sont «immatures » et qu'ils présentent une plus grande micro-hétérogénéité que ceux des STF dont ils diffèrent totalement.

\section{ROLE DES GLYCANNES DANS LES FONCTIONS BIOLOGIQUES DES TRANSFERRINES}

En vue de préciser la participation des glycannes dans les fonctions biologiques des transferrines, nous avons déglycosylé la sérotransferrine de Lapin à l'aide d'une endo-N-acétyl- $\beta$-D-glucosaminidase récemment isolée au Laboratoire à partir de cultures de Basidiomyces (Bouquelet et al., 1980). 
$\operatorname{NeuAc}(\alpha 2-6) \operatorname{Gal}(\beta 1-4) \operatorname{GlcNAc}(\beta 1-2) \operatorname{Man}(\alpha 1-3)$

$\operatorname{NeuAc}(\alpha 2-6) \operatorname{Gal}(\beta 1-4) \operatorname{GlcNAc}(\beta 1-2) \operatorname{Man}(\alpha 1-6) / \operatorname{Man}(\beta 1-4) \mathrm{GlcNAc}(\beta 1-4) \mathrm{GlcNAc} \beta 1-$ Asn

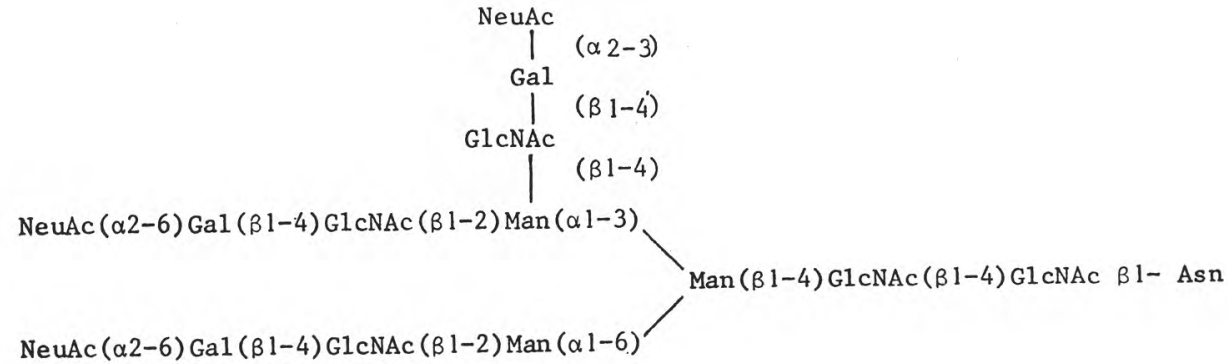

fig. 1

Structure des glycannes bi et triantennés de la sérotransferrine humaine.

Structure of the bi and triantennary glycans of human serum transferrin.

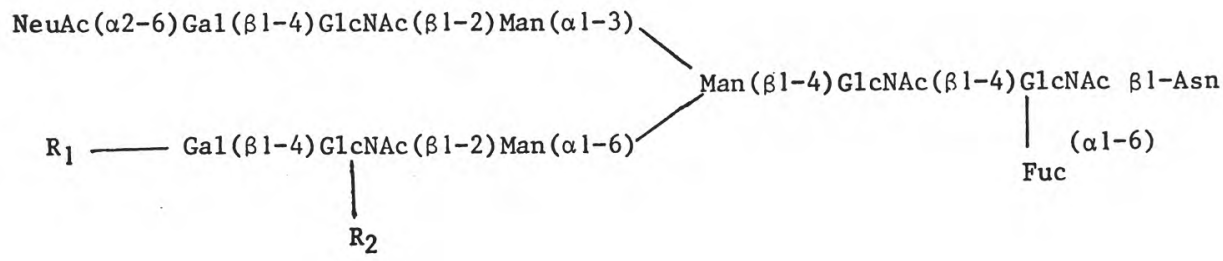

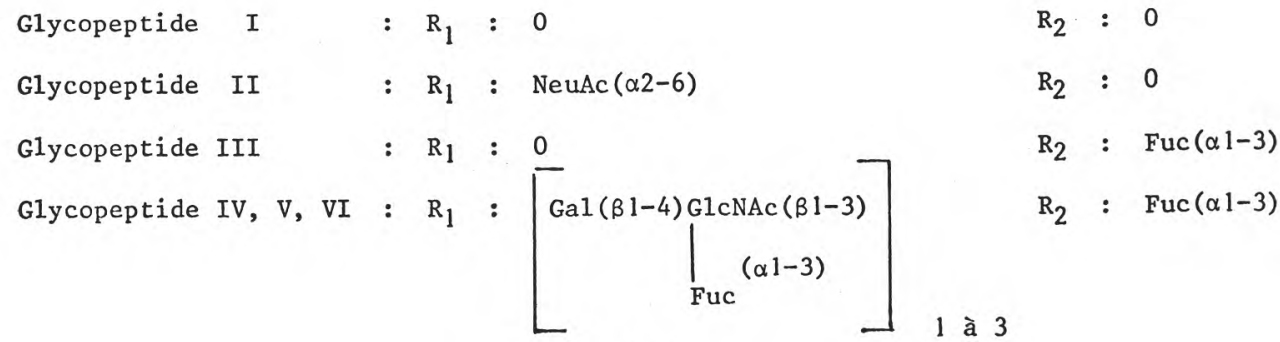

fig. 2

Structure des glycannes de la lactotransferrine humaine.

Structure of the glycans of human lactotransferrin. 


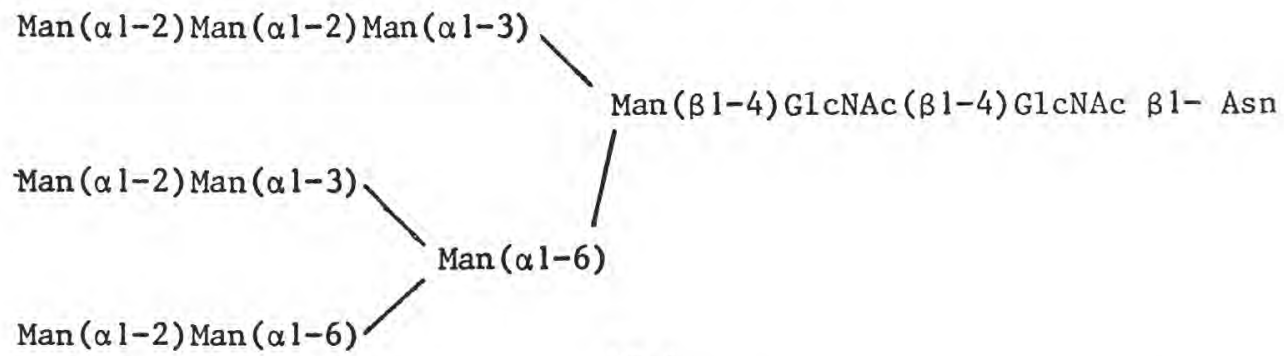

fig. 3

Structure du glycanne oligomannosidique majeur de la lactotransferrine de vache.

Structure of one oligomannosidic glycan from bovine lactotransferrin.

Nous avons pu de cette manière constater que la déglycosylation ne modifiait en rien la capacité de fixation réversible de 2 ions $\mathrm{Fe}^{+3}$ par la sérotransferrine, pas plus que l'association de cette dernière aux membranes des réticulocytes. Le passage lui-même du ${ }^{59} \mathrm{Fe}$ dans les réticulocytes n'est nullement altéré comme en témoigne l'isolement d'hémoglobine contenant du fer radioactif (Léger et al., 1982). La non-intervention des glycannes des sérotransferrines dans la reconnaissance des récepteurs réticulocytaires explique qu'ils possèdent des structures primaires identiques quelle que soit l'espèce dont la sérotransferrine tire son origine.

$\mathrm{Au}$ contraire, dans le cas de la lactotransferrine humaine, les groupements glycanniques peuvent intervenir dans le mécanisme complexe de la défense antibactérienne de l'intestin par cette glycoprotéine. En effet, Izhar et al., 1982 et Pierce-Cretel, Mirelman, Montreuil et Spik (résultats non publiés) ont démontré que des glycopeptides de la lactotransferrine inhibaient la reconnaissance des membranes de cellules intestinales par les lectines de Shigella.

Toutefois, en dépit de ce dernier résultat, reste posée la question de savoir pourquoi les transferrines - les sérotransferrines, en particulier — sont glycosylés.

\section{Bibliographie}

Bouquelet (S.), Strecker (G.), Montreuil (J.) and Spik (G.) (1980). Characterization of a novel endo-N-acetyl- $\beta$-D-glucosaminidase from the culture filtrate of a Basidiomycete (Sporoticum dimorphosporum), active on biantennary mono- and asialoglycoasparagines of the $\mathrm{N}$-acetyllactosaminic type. Biochimie, 62, 43-49.

Izhar (M.), Nuchamowitz (Y.) and Mirelman (D.) (1982). - Adherence of Shigella flexneri to Guinea pig intestinal cells is mediated by a mucosal adhesin. Infec. Immunity, 35, 1110-1118. 
Jeltsch (J. M.) and Chambon (P.) (1982). - The complete nucleotide sequence of the chicken ovotransferrin mRNA. Eur. J. Biochem., 122, 291-295.

Léger (D.), Tordera (V.), Spik (G.), Dorland (L.), HaVerkamp (J.) and Vliegenthart (J. F. G.) (1978). - Structure determination of the single glycan of rabbit serotransferrin by methylation analysis and $360 \mathrm{MHz}{ }^{1} \mathrm{H}-\mathrm{NMR}$ spectroscopy. FEBS-Lett., 93, 255-260.

Léger (D.), Michel (V.), Montreuil (J.) and SpiK (G.) (1982). - Properties of deglycosylated rabbit transferrin. Biochim. Biophys. Acta (à paraître).

Mac Gillivray (R. T. A.), Mendez (E.), Sinha (S. K.), Sutton (M. R.), LinebackZINS (J.) and BREW (K.) (1982). - The complete amino-acid sequence of human serum transferrin. Proc. Nat. Acad. Science U.S.A., 79, 2504-2508.

Metz-Boutique (M. H.), Mazurier (J.), Jollès (J.), Spik (G.), Montreuil (J.) and Jollès (P.) (1981). - The present state of the human lactotransferrin sequence. Study of the alignment of the cyanogen bromide fragments and characterization of N and C terminal domains. Biochim. Biophys. Acta, 670, 243-254.

Spik (G.), Bayard (B.), Fournet (B.), Strecker (G.), Bouquelet (S.) and Montreuil (J.) (1975). - Studies on glycoconjugates. LXIV. Complete structure of two carbohydrate units of human serotransferrin. FEBS-Lett., 50, 296-299.

Spik (G.), Strecker (G.), Fournet (B.), Bouguelet (S.), Montreuil (J.), Dorland (L.), van Halbeek (H.) and Vliegenthart (J. F. G.) (1982). - Primary structure of the glycans from human lactotransferrin. Eur. J. Biochem., 121, 413-419.

Spik (G.), Debruyne (V.), Montreuil (J.), Dorland (L.), van Halbeek (H.), and VliegenthaRT (J.F.G.) (1982). - Complete structure of the triantennary glycans of human serum transferrin. FEBS-Lett. (à paraître).

Van Halbeek (H.), Dorland (L.), Vliegenthart (J. F. G.), Spik (G.), Chéron (A.) and Montreuil (J.) (1981). - Structure determination of two oligomannosidic type glycopeptides obtained from bovine lactotransferrin, by $500 \mathrm{MHz}{ }^{1} \mathrm{H}-\mathrm{NMR}$ spectroscopy. Biochim. Biophys. Acta, 675, 293-296.

Williams (J.), Elleman (T. C.), King (A. G.) and Kuhn (K. A.) (1982). - The primary structure of hen ovotransferrin. Eur. J. Biochem., 122, 297-303. 